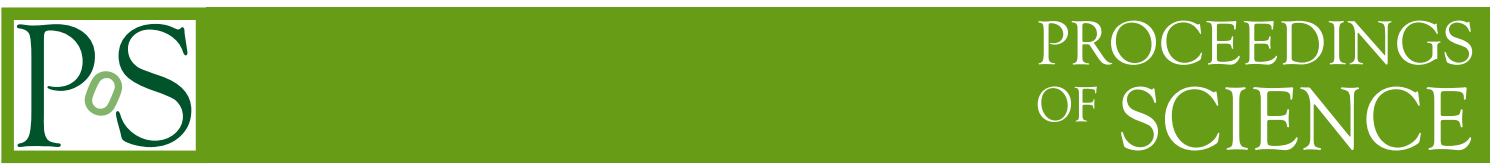

\title{
Perseus anomalous emission as seen by VSA
}

\author{
Robert Watson* ${ }^{* \dagger}$ \\ Jodrell Bank Observatory, University of Manchester \\ E-mail: rawliac.es \\ R. Rebolo, ${ }^{a}$ R.D. Davies, ${ }^{b}$ R.J. Davis ${ }^{b}$ and J.A. Rubiño-Martin ${ }^{a}$ \\ ${ }^{a}$ Instituto Astrofisica Canarias (IAC), Spain. \\ ${ }^{b} J B O$, University of Manchester, United Kingdom.
}

\begin{abstract}
We have observed the Perseus Molecular cloud at $33 \mathrm{GHz}$ at 7 arcminute resolution with the Very Small Array (VSA), which was previously identified as a strong source of anomalous dust correlated microwave emission at $1^{\circ}$ with the COSMOSOMAS experiment. This emission represents a relatively new and poorly understood galactic foreground which may be significant at intermediate galactic latitudes. The angular resolution of the VSA makes it ideal for comparison with the IRAS satellite survey and is similar to that of the HFI channels of the forthcoming Planck mission. The morphology of this emission at $33 \mathrm{GHz}$ consists of several 10 arcminute hot-spots coincident with the IRAS 'knots' in G159.6-18.5 plus a strange decrement, which is probably a hole in the diffuse anomalous component. We find the IRAS $25 \mu \mathrm{m}$ data the most correlated template at $77 \%$ using the Pearson correlation statistic.
\end{abstract}

$C M B$ and Physics of the Early Universe

20-22 April 2006

Ischia, Italy

\footnotetext{
* Speaker.

'Currently at the IAC.
} 


\section{Introduction}

In Watson et al. (2005) [12] we identified a bright source of correlated dust emission G159.618.5 in the Perseus molecular cloud, where a strong correlation is seen between far-infrared DIRBE $100 \mu \mathrm{m}$ and microwave COSMOSOMAS/WMAP data, but with no corresponding radio signal which could explain it as associated free-free or synchrotron emission. Similar effects have been seen by Davies et al. (2005) [3] suggesting that anomalous emission is significant at intermediate galactic latitudes. The properties of this region have been studied in detail by Andersson et al (2000) [1] and Ridge et al [10]. Fitting for the flux of Perseus defines its spectral energy distribution (SED) showing a component peaking at $42 \mathrm{Jy}$ at $22 \mathrm{GHz}$ which can be fitted with electric dipole emission from rapidly spinning small dust grains (aka 'spinning dust' Draine and Lazarian 1998 [4]). Further COSMOSOMAS observations at $11 \mathrm{GHz}$ have made polarisation measurements of Perseus (Battistelli et al. 2006 [目]) have placed an upper limit of 3\% on polarization. If anomalous emission is polarised it will become a major contaminant for future polarisation experiments. Looking for anomalous emission on smaller scales have proved to be more difficult but with some detections (Finkbeiner et al. 2002[8], Finkbeiner 2004[9], Casassus et al. 2004[5] and Casassus et al. 2006[6]). It has even been suggested a grain segregation model where the emission does not follow the $100 \mu \mathrm{m}$ distribution but more with the higher frequencies where transiently heat small dust grains are more important. (Oliveira-Costa et al. 2002[7]). It therefore makes sense to look on smaller scales where there is known to be a signal found at lower resolution to test this hypothesis.

\section{The Very Small Array}

The Very Small Array is a radio interferometer designed to measure the CMB angular power spectrum, built and run by MRAO (Cambridge), JBO (Manchester) and IAC (Tenerife) and funded by PPARC. It has recently been upgraded new receivers and with larger $65 \times 90 \mathrm{~cm}$ lightweight carbon-fibre antennas allowing the utilisation of the whole of the $4 \times 3 \mathrm{~m}$ support table area for longer baselines in 'super-extended' configuration. This produces a synthesised beam of 7 arcminutes resolution comparable to the Planck HFI channels, but at $33 \mathrm{GHz}$. Since this is also the resolution of the IRAS survey data, this makes VSA ideal to check for the correlation of regions of anomalous emission with FIR dust emission at this angular scale.

Perseus was included as part of the VSA foreground assessment program of pointed observations of dust rich fields. Due to its size $\left(2 \times 1^{\circ}\right)$ Perseus has to be covered by a mosaic of 9 pointings of the VSA $1^{\circ}$ primary beams. Each pointing has had 10-15 hours of observation and these have been processed by the normal VSA analysis software for editing and calibration. The CRAB radio source, which is 26 degrees away was used as the flux and phase calibrator.

The VSA map was produced by phase rotating all visibilities to the same phase centre in the middle of the central field and then CLEANed within AIPS. The VSA $33 \mathrm{GHz}$ map is shown as contours on top of the $100 \mu \mathrm{m}$ IRAS map in Fig. 1a. The use of the AIPS CLEAN boxes has also shown the northern decrement to be real and not an artifact. The next section quantifies the degree of correlation between FIR and VSA. 


\section{Correlation analysis}

The objective of correlation analysis is to establish the coupling constants to various templates to help understand the emission mechanisms involved and help predict foreground contamination by extrapolation to other regions. Also the degree of correlation with a particular template helps decide the source of the emission and how well it is traced. For example anomalous emission is suspected to come from spinning very small grain (VGR) dust and therefore should correlate more with the shorter wavelength IRAS bands where the proportion of VSG to large gain dust emission is higher. This can be quantified by the Pearson correlation statistic as used by Casassus et al. (2006)[6] in the dark cloud LND 1622.

Fig. 1a shows contours from a VSA CLEAN mosaic map on top of the IRAS $12 \mu \mathrm{m}$ map, where there is clearly a high degree of correlation apart from IC348, which is much weaker than expected. Looking at the dust temperature maps of Schnee et al. (2005) [COMPLETE project][11], reveal IC348 and a region inside the ring to be due to warm dust rather than the cooler dark cores to which the VSA emission seems to correspond. We therefore remove two 24 arcminute diameter areas from the FIR maps which correspond roughly to regions above $20 \mathrm{~K}$.

To carry out the correlation the IRAS maps have to be filtered to allow for the flux loss inherent in the interferometer response due to the sampling of the U-V plane. We do this by simulating each VSA pointing on the FIR maps allowing for the primary beam of 1 degree, Fourier transforming and sampled with the same points in the U-V plane. We apply phase rotation to a common phase point and sum the pointings to produce a dirty FIR map. The two masked regions must be applied before the Fourier transform so as not to contaminate the rest of the map. The dimensionless coupling constant is normalised relative to $33 \mathrm{GHz}$ and is produced by the linear correlation of the two pixels sets. At the same time the Pearson correlation statistic is produced. We have also included the MSX $8.3 \mu \mathrm{m}$ data to extend the wavelength range (available from NASA/IPAC Infrared Science Archive) by passing it through the AIPS task HGEOM to match the IRAS fits data. How the correlation statistic varies with frequency is shown in Fig. $1 \mathrm{~b}$ for both the masked and unmasked cases.

\section{Conclusions}

Warmer cores hotter than $20 \mathrm{~K}$, which dominate the IRAS data are relatively weak in anomalous emission and have been masked. The masked templates achieve a high degree of correlation peaking in the $25 \mu \mathrm{m}$ map with $77 \%$ correlation, this may be due to molecular line contamination which the shortest wavelenght bands suffer. The same spectral response is found compared to the COSMOSOMAS emission confirming these VSA 'knots' have the same characteristics. The northern decrement may prove more interesting as it may point to the mechanism driving the anomalous emission and why its emissivity varies so much from cloud to cloud. As yet there still no viable way to generate a template for anomalous emission.

\section{References}

[1] Andersson, B-G.,Wannier, P.G., Moriarty-Schieven, G.H. and Bakker, E.J. 2000, AJ, 119, 1325 

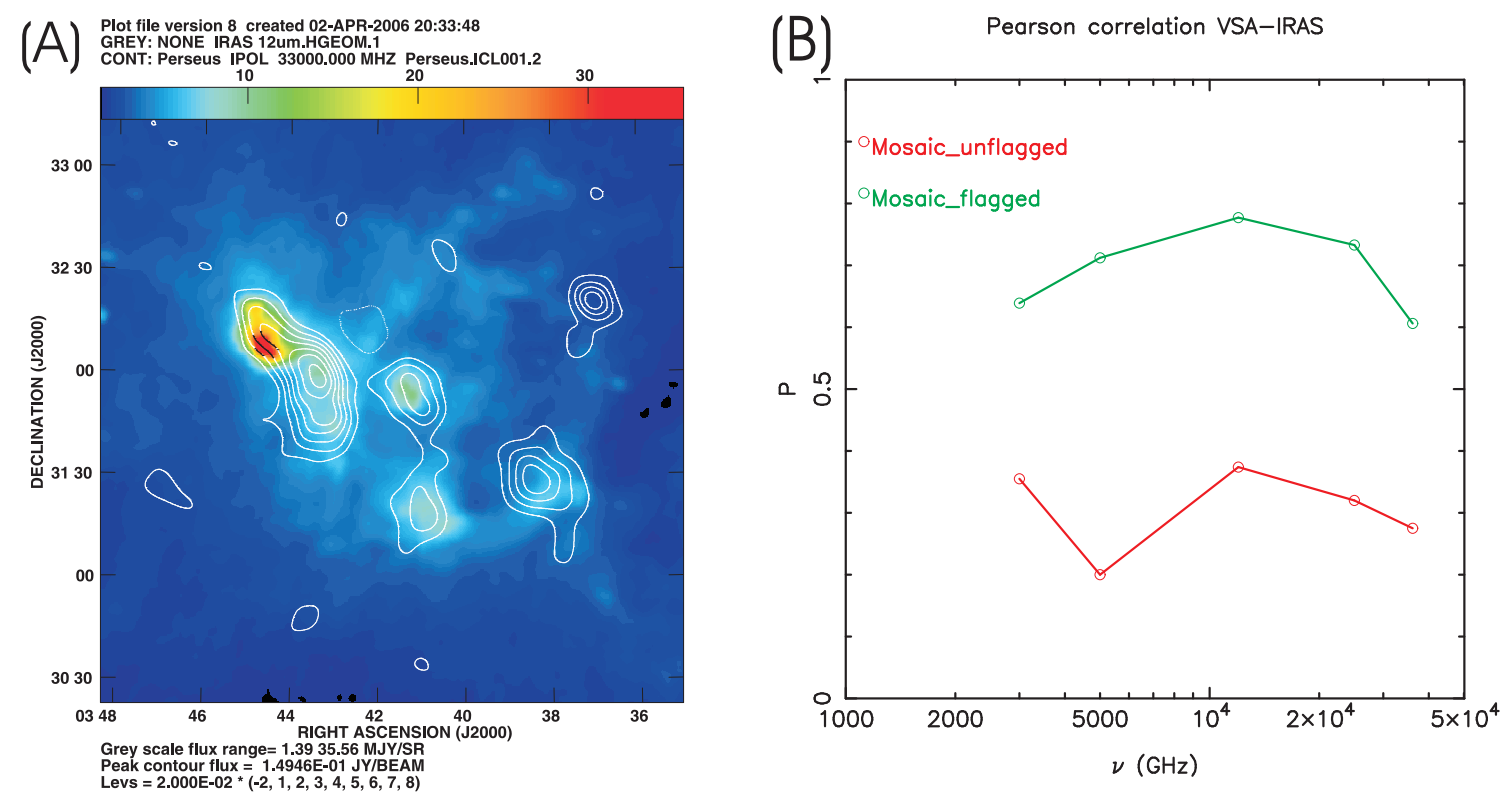

Figure 1: (a) Contours from the VSA CLEAN map overlaid on the IRAS $100 \mu \mathrm{m}$ map of the Perseus cloud showing the alignment of VSA features and IRAS cores. (b) The Pearson correlation coefficients between the VSA dirty map and filtered IRAS and MSX maps with and without masking of the warmer cores.

[2] Battistelli, E. S., Rebolo, R., Rubiño-Martín, J. A., Hildebrandt, S. R., Watson, R. A., Gutiérrez, C., Hoyland, R. J. 2006, accepted ApJ [astro-ph/0603379]

[3] Davies, R. D., Dickinson, C., Banday, A. J., Jaffe, T. R., Gorski, K. M., Davis, R. J. 2005 submitted [astro-ph/0511384]

[4] Draine, B.T. and Lazarian, A. 1998, ApJ, 508, 157

[5] Casassus,S. Readhead, A.C.S., Pearson, T.J., Nyman, L.A., Shepherd, M.C., Bronfman, L. 2004, ApJ, 603,599

[6] Casassus, S., Cabrera, G. F., Förster, F., Pearson, T. J., Readhead, A. C. S., Dickinson, C. 2006 ApJ, 639,951

[7] de Oliveira-Costa, A., et al. 2002, ApJ, 567, 363

[8] Finkbeiner, D.P. Schlegel, D.J., Frank, C., Heiles, C. 2002, ApJ, 568, 898

[9] Finkbeiner, D.P. 2004, ApJ, 614, 186

[10] Ridge, N.A., Schnee,S.L., Goodman, A.A., Foster, J.B. 2006, [astro-ph/0601692]

[11] Schnee, S.L., Ridge, N.A., Goodman, A.A., Li, J.G. 2005, ApJ, 634, 442

[12] Watson, R. A., Rebolo, R., Rubiño-Martin, J. A.; Hildebrandt, S., Gutiérrez, C. M., Fernández-Cerezo, S., Hoyland, R. J., Battistelli, E. S. 2005, ApJ, 624, L92 